\title{
BIM for existing facilities: feasibility of spectral image integration to 3D point cloud data
}

\author{
Kinjiro Amano ${ }^{1, a}$ and Eric Lou ${ }^{1}$ \\ ${ }^{1}$ School of Mechanical, Aeroplane and Civil Engineering, University of Manchester, Sackville Street, Manchester M13 9PL, UK
}

\begin{abstract}
Accurate geometrical and spatial information of the built environment can be accurately acquired and the resulting 3D point cloud data is required to be processed to construct the digital model, Building Information Modelling (BIM) for existing facilities. Point cloud by laser scanning over the buildings and facilities has been commonly used, but the data requires external information so that any objects and materials can be correctly identified and classified. A number of advanced data processing methods have been developed, such as the use of colour information to attach semantic information. However, the accuracy of colour information depends largely on the scene environment where the image is acquired. The limited number of spectral channels on conventional RGB camera often fails to extract important information about surface material, despite spectral surface reflectance can represent a signature of the material. Hyperspectral imaging can, instead, provide precise representation of spatial and spectral information. By implementing such information to 3D point cloud, the efficiency of material detection and classification in BIM should be significantly improved. In this work, the feasibility of the image integration and discuss practical difficulties in the development.
\end{abstract}

\section{Introduction}

The UK will be required to refurbish or retrofit $93 \%$ of existing buildings by 2050 to meet national carbon targets - this includes 25 million homes and 3 million nondomestic facilities [1]. Refurbishing and retrofitting (RR) facilities will be the major work for the UK construction industry in the next 20 years. The main issue of RR is the unavailability or inaccurate building drawings, plans or blueprints [2]. Without these, the RR project is open to a myriad of engineering unknowns and risks, causing additional concerns to health and safety, financial uncertainties and hazards. To optimise RR, any project team must be able to work seamlessly with accurate building information (e.g. plans, material) on a digital and common platform called Building Information Modelling (BIM) [3, 4]. BIM is a digital representation of the physical and functional characteristics of a buildings or structures (hereby referred to as facility). It is aimed to serve as a shared knowledge resource for information about a facility, as well as forming a reliable basis for decisions during the facility's lifecycle, from inception onward. BIM has been becoming essential tool for the Architecture, Engineering, Construction and Facility Management communities. The use of BIM is also mandated for UK Government projects by 2016 [5].

BIM allows accredited project team members to add, edit, delete, and share real time information and design specification on a common technology. BIM provides multi-dimensional information of any facility; identify design collision between the multidisciplinary project team; check for accessibility compliances; capture design and material information; building regulation compliance; supply chain integration [6-8].

Among these information, geometrical information of the facilities and objects, and their physical properties must be documented accurately so that the digitised information can be linked together on a common standard platform.

Technological advancements have made it possible to generate 3D models to assess as-built conditions, and to verify the progress of the project and contract specifications. Modelling can be used to capture the asbuilt or as-is reality of any project and facility. The modelling consists of collecting infrastructure's spatial data as a series of 3D Cartesian coordinates. The collection of the dense Cartesian-based distance data set is known as point cloud, and these points are processed and transformed into a structure or an object by purposely designed software [e.g. 9] associated with specific database [e.g. 10].

The 3D laser scanning and photogrammetry have been most commonly used to acquire the point cloud data $[11,12]$. Developments in both hardware and software have been underway to make them more reliable, compact, and affordable, but there are still certain limits in the implementation of the 3D point cloud to BIM. One of the critical issues is to do with performing identification and classification of objects and materials in the $3 \mathrm{D}$ point cloud.

\footnotetext{
${ }^{a}$ Corresponding author: kinjiro.amano@manchester.ac.uk
} 
The point cloud data does not contain any sematic information but merely geometrical coordinates and intensity values per point. This problem can be addressed by combining external information, such as visual information from the other sources, to the point cloud. The most common method is the integration with snapshot photographs or colour images taken independently of the laser scanning but simultaneously on site [13-15]. One of various developments in the utilisation of colour information processes colour images with sophisticated algorithm along with specifically designed image database to identify the construction materials [16].

A concern is, however, the quality of the colour information by conventional RGB camera depends on the environment or imaging condition where the images are being taken. Colour appearance of the surfaces, for example, varies with the scene illumination. In addition, due to the limited number of the spectral channels in the imaging system, any complex spectral signature of materials cannot be accurately extracted. These disadvantages lead to failures in the identification and classification.

Since the identification of the materials can be provided by their spectral signature, it would be ideal if the spectral imaging data is integrated to $3 \mathrm{D}$ point cloud data. With the current technology, however, the hyperspectral imaging is available in two dimensions. A challenge is, therefore, to develop methodology to integrate the 2D spectral image to the 3D point cloud. One of feasible and convenient methods is to project the $3 \mathrm{D}$ point cloud to $2 \mathrm{D}$ and apply the image registration methods between 2D images. In this report, the feasibility of this integration to the hyperspectral data is addressed, and the advantages and disadvantages are to be discussed.

\section{Colour image for material identification}

The laser scanning is one of the most common methods to capture geospatial information in the built-environment and facilities accurately and efficiently. Photogrammetry can be used complementary because of the similarities in output data [11].

The point cloud, as noted earlier, only contains Cartesian coordinate and intensity values at the point, and does not carry any semantic information, such as knowing which point belongs to what as-built structural component. Without such attributes or context, it is almost impossible to judge where the point is belonged to and to examine any relationship between nearby points. In order to identify and classify the materials and objects in the facilities or scenes, it is necessary to attach additional semantic information to the point cloud.
Working with such featureless data to explore geometric reasoning are generally tedious, time consuming, often produce errors [17].

The semantic information or context of the scene can be extracted from independent imaging systems, preexamined data, or post image processing [18, 19]. A number of computational algorithms have been also reported to provide better solutions on these limitations [e.g. 13, 14, 16, 18].

Colour information and photographic data often provides useful information about the objects in the facilities or scenes, and even what the materials are. The colour images taken by conventional RGB camera have an advantage to be collected quickly and inexpensively. For example, Dimitrov et al [14] presented a new algorithm based on Structure-from-Motion (SfM) combined with image statistical analysis. Hang et al [16] proposed a manual integration of 3D point cloud and 2D image patches to facilitate 4D BIM with the site photo$\log$ and SfM accompanied with specially designed image database.

However, the colour imaging by a conventional RGB camera has certain limitations. That is, the quality of the colour largely defined by the environmental condition where the image is being acquired. Colour appearance of the surfaces, for example, is influenced by the scene illumination.

Figure 1 shows an example of misrepresentation of colours. Colour images were acquired by a build-in RGB camera in a laser scanner (Fig. 1a), and by a conventional RGB camera (Fig. 1b). Despite the materials of the all surfaces are the same, top surface has a different appearance from the side surfaces (Fig. 1a) and brightness of the surfaces are different between the images (Fig. $1 \mathrm{a}$ and b). Further, due to the limited number of the spectral channels over spectrum (three in the conventional RGB camera and human eye), such imaging device cannot always produce correct colour appearance of some surfaces which has complex spectral properties are, known as metameric surfaces [20]. (a)

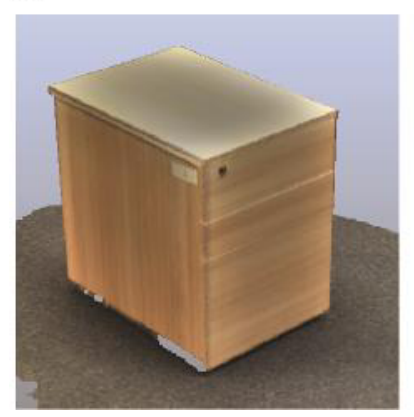

(b)

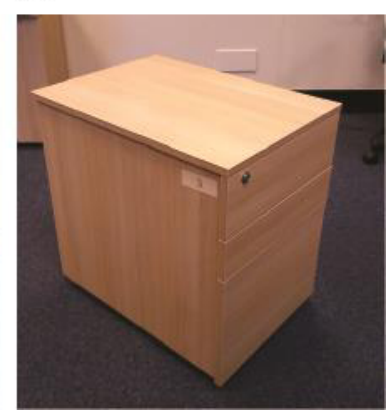

Figure 1. Examples of misrepresentation of colour appearance. Colour images taken by (a) the built-in RGB camera in the laser scanner and by (b) an external conventional RGB camera. 
(a)

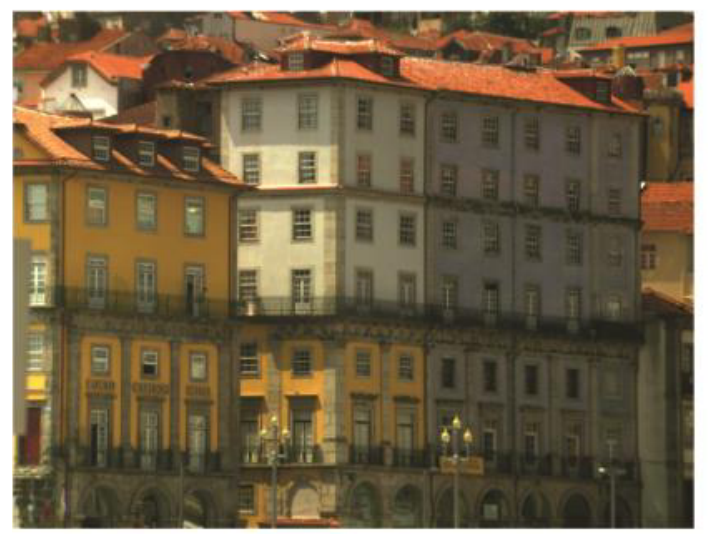

(c)

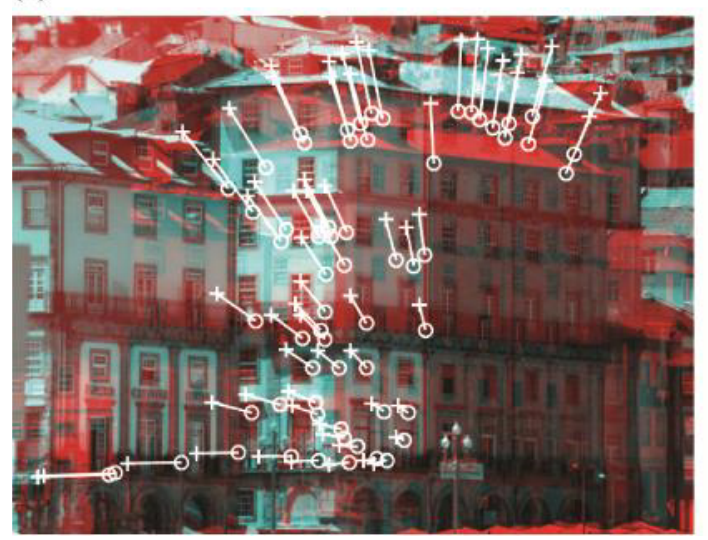

(b)

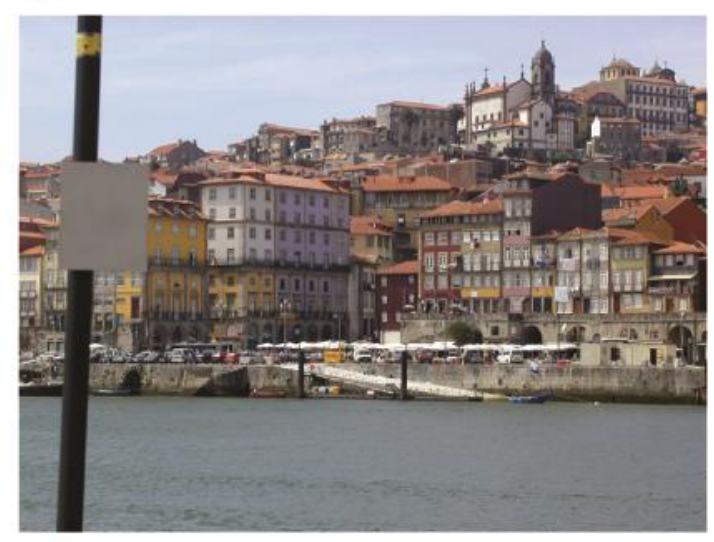

(d)

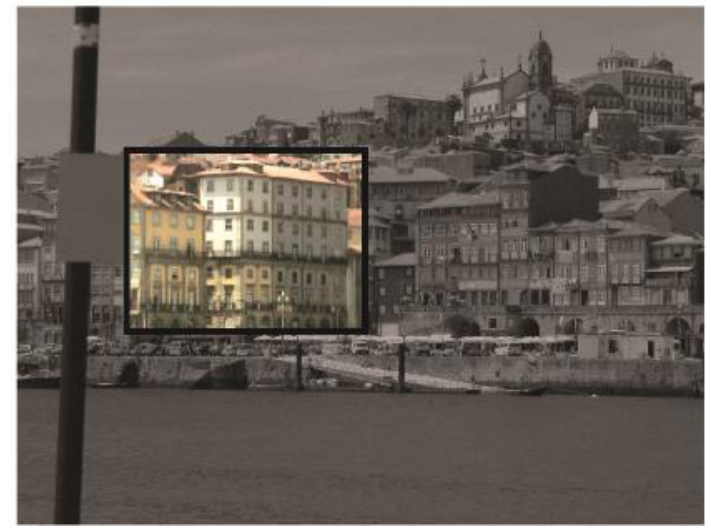

Figure 2. The image registration example between two images acquired by different conditions (in spatial resolution, viewing distance and viewing). (a) Colour rendering image based on the hyperspectral imaging in a natural scene. (b) Colour image of the same scene with a conventional RGB camera. (c) Detection of the SURF (Speeded-Up Robust Features) image key features and image transformation. Circles and crosses are the features from each of the images in (a) and (b), respectively. (d) Result of image registration. For visibility a black frame was inserted and the background was converted to grey scale.

\section{Advanced imaging and integration}

\subsection{Spectral information}

Characteristics of materials can be represented by spectral properties. Estimation of surface reflectances enables to identify the spectral characteristics of materials independently of scene illuminations and environment. The more spectral channels are available, the more accurate estimation is available. It is therefore reasonable to consider the integration of the hyperspectral imaging data to 3D point cloud.

Spectral characteristics of urban construction materials, such as an aging effect on concrete and clay tiles, have been examined to establish spectral library for the BIM [21]. It has been also the case for the spectral characteristics of asphalt road aging and deterioration [22]. These data provide the important evidence that the spectral characteristics and their examination need to be taken into account to identify the materials, and consequently to be implemented in the BIM protocol. They also indicate the requirement of a careful consideration to determine which range of spectra should be examined.

\subsection{Hyperspectral imaging}

The hyperspectral imaging has evolved to include not just one monochromatic channel or three colour channels covering the visible spectrum, but many channels, over 30 , even several hundreds, extending from the visible spectrum to near-infrared (NIR) and shortwave infrared (SWIR) channels [20, 23].

This imaging method is aimed mainly to exploit the materials comprising the various objects in a scene where lights reflect, scatter, and being absorbed, depending on characteristic of material in both chemical and physical properties.

If the number of spectral channels sufficiently covers a wider spectral range and each is narrowly tuned, the information on each pixel can be used in a detailed analysis to gain accurate identification of objects and materials against different environmental exposure. Such assessment is unavailable by human eyes or the RGB camera with the three channels.

In the recent years, demands to the hyperspectral imaging has grown significantly because of its increasing applications in a variety of research area, for example, agriculture [24] and cultural heritage [25]. The hyperspectral imaging is currently limited to two 
dimensions and requires specific spectral calibration at each image acquisition. Each spectral channel has different focal length. This causes chromatic fringes in the resulting images, unless any spectral correction is made by performing post-image processing (e.g. the image registration across the spectral channels) or by using any optical devices in the imaging system (e.g. an achromatising lens).

\subsection{Image registration methods}

One of the unique features of using the 3D point cloud data is the ability to manipulate and view data in a full degree of freedom environment. That is, users can interact and manipulate the dense point data flexibly (for example, users can observe the data from different distance and viewing angles as shown in Fig. 3a), allowing for construction of as-built conditions in a virtual environment [26]. Such flexibility may provide a feasibility of integrating the point cloud to the other digital imaging. That is, it is possible to adjust the viewing angle so that the projection of the $3 \mathrm{D}$ image can closely correspond to the external 2D image. The projected 2D image can be registered to the external 2D image to be integrated. It enables to extract or attach additional information.

Once both image data from the laser scanning and hyperspectral imaging are on the same image dimension, the integration will be performed by applying the image registration methods established in computational vision. Common technique in the image registration is based on the detection of image key features and their correspondences between the images. The key features are, for example, intensity contrast, gradients, edge, and surface normal. Several algorithms have been proposed to detect the key features; Binary Robust Invariant Scalable Keypoints (BRISK) [27]; Speeded-Up Robust Features (SURF) [28]; Maximally Stable Extremal Regions (MSER) [29]; Fast Retina Keypoint (FREAK) [30]. The combination of these methods is also applicable. Instead of using computational key features, it is also possible to use reference objects or markers physically placed in the scenes. These image registration algorithms are applied to the $3 \mathrm{D}$ point cloud as well as 2D images, when multiple sets of scanning (point clouds) are to be registered [31].

Figure 2 represents an example of the image registration between two images taken by the different imaging systems, from different viewing distances and angles, with applying the SURF algorithm [28] to compute the image transformation (Fig. 2c). The goodness of the image registration is controlled by adjusting optimisation parameters, such as the number of iterations and step size. The final registered image is shown in Fig. 2 d. (a)

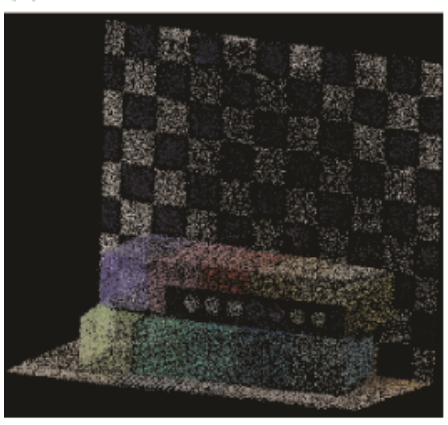

(b)

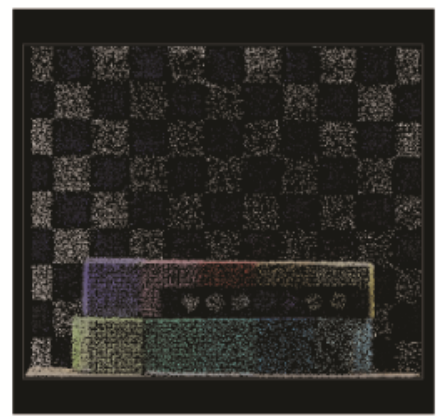

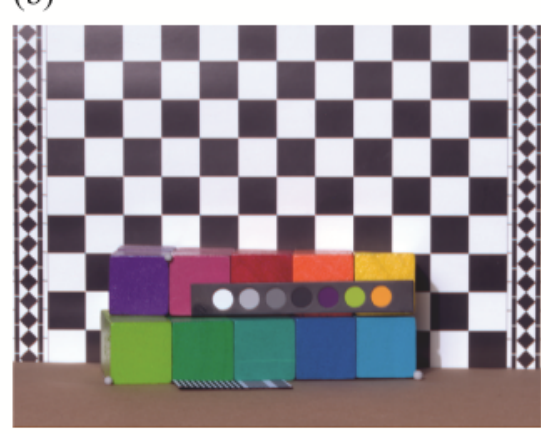

(c)

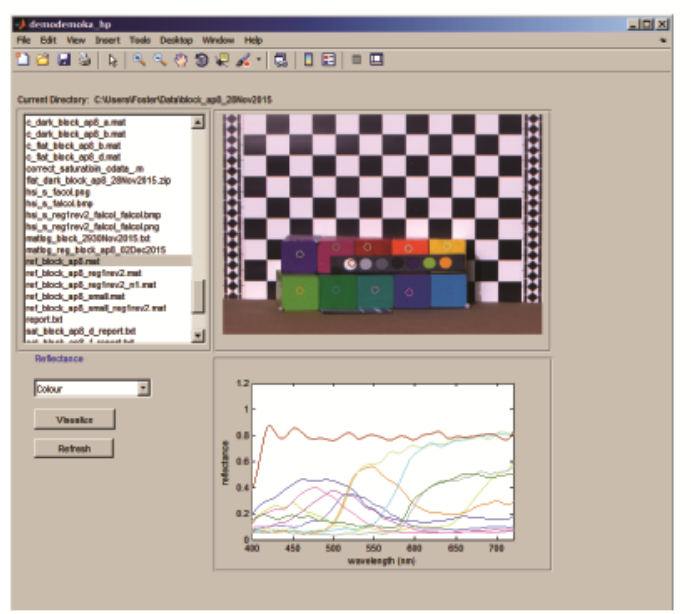

Figure 3. Proposed image integration between 3D point cloud and the hyperspectral image. (a) Point cloud with adjustable viewing angle; (b) Colour image rendering of the hyperspectral data. (c) Example GUI to be implemented to monitor spectral profile on each pixel selected. 
If the image integration is completed, the spectral properties obtained by the hyperspectral data (as shown in Fig. 3c) can be integrated to point cloud. The integration between $3 \mathrm{D}$ point cloud and spectral images have been reported in the other research disciplines, for example, characterisation of plants and plant disease detection [24, 32] and examination of art-objects [25], any of which have been performed under well-controlled experimental environment.

\subsection{Considerations}

This proposed method is not difficult to implement, but it should be noted that there are certain disadvantages.

The projection to $2 \mathrm{D}$ loses the advantage of having 3D information, such as depth and occlusion. Any of the image information on the invisible surfaces cannot be obtained. It will be problematic if any significance (e.g. damage) may exist on those surfaces. The $3 \mathrm{D}$ information also provides useful information about the effect of scene illumination and shadows, which are often important in designing and retrofitting of the built-environment and facilities. It is, therefore, a common practice to identify what image information will be required and examine the geometrical correspondence between different imaging techniques in advance of performing the image acquisitions.

Difference in spatial resolution of the imaging devices may cause inaccuracy in the image registration. Advanced image processing tools may be applied to achieve sufficient interpolation or extrapolation to overcome this issue.

In terms of spectral image data, as noted earlier, physical characteristics of light must be taken into account, namely, chromatic aberration. The fact that each spectral channel (light of different wavelength) has different focal length causes "colour fringe" at the edge of surfaces in the image. The correction of the chromatic aberration is important because edges of surfaces and objects provide essential information in their identification and classification. The correction is available at the time of image acquisition using specially designed optical devices (e.g. an achromatising lens) or in the post image processing (e.g. the image registration across the spectral channels). As a note, there has been a report that the spectral edges or gradient of the spectral map provide clue to achieve image fusion [33].

There is a technical disadvantage in the image registration by image key features. That is, the detection of any key features depends on the image contents. If the scene is consisted of, for example, a large uniform surface or walls, it is difficult to detect any key features. Alternative registration algorithm, such as normal cross correlation may be considered.

Apart from the proposed image integration method, the computational image fusion methods between 3D point cloud and 2D colour image have been reported [34], where the characterisation of the imaging devices is implemented. The camera calibration is considered as a necessary step in the 3D computer vision to extract spatial and geometrical information from 2D images [35,
36]. It is the process of estimating intrinsic and extrinsic parameters. Intrinsic parameters deal with the internal characteristics of the imaging device, such as, focal length, skew, distortion, and image centre. Extrinsic parameters describe its position and orientation in the world coordinate. Knowing these parameters is essential, as it allows estimating the scene's structure in Euclidean space and removes lens distortion, which degrades accuracy. Such characterisation of the devices should be performed ahead of the imaging practice. It still needs, however, to examine exactly how the correction works under practical and uncontrolled environments.

\section{Conclusions}

Refurbishment and retrofit (RR) projects will be the main work in the UK in order to achieve the agreed carbon emission targets. RR projects is not plagued with risks and uncertainties, which considerably rises costs, health and safety, and lacked environmental and carbon emission consideration. It has been considered that BIM for RR projects is the way forward but has not been fully practiced in industries yet. The 3D scanning of existing facilities to obtain spatial information has been introduced and the demand has been increasingly grown. Taking a step further, the present report considered a possible method of the image integration between the $3 \mathrm{D}$ point cloud and 2D hyperspectral imaging. As discussed, there are considerable difficulties at this stage of the development. Some of the disadvantages will be overcome by careful pre-planning of the imaging protocol. Currently computational cost for the point cloud and the spectral data is very demanding. Developments in sophisticated image processing tools are also required to extract all of the relevant information from the multitude of the data. However, by achieving the image integration between $3 \mathrm{D}$ and $2 \mathrm{D}$, the identification and classification of the construction materials will become more efficient in terms of both labour and cost. This approach will add another dimension to the BIM.

\section{Acknowledgement}

This work was supported by British Council NewtonUngku Omar Fund (No.172726659).

\section{References}

1. J. Edwards and A. Townsend. Buildings under refurbishment and retrofit, Carbon Action 2050 White papers, The Chartered Institute of Building (2011)

2. C. Gorse and D. Highfield Refurbishment and Upgrading of Buildings. Abingdon, UK, Spon Press (2009)

3. D. Bryde, M. Broquetas and J. M. Volm, International Journal of Project Management 31, 7 (2013)

4. BIS. Building information modelling, Department for Business, Innovation \& Skills, HMGovernment, UK (2012) 
5. Cabinet Office. Government Construction Strategy, Cabinet Office, UK (2011)

6. R. Howard and B.-C. Björk, Adv. Eng. Inform. 22, 2 (2008)

7. Y. Arayici, Structural Survey 26, 3 (2008)

8. K. Barlish and K. Sullivan, Autom. Constr. 24 (2012)

9. Autodesk. Building Information Modeling for Sustainable Design Autodesk (2005)

10. RIBA Enterprises. NBS National BIM Library (2016)

11. S. El-Omari and O. Moselhi, Autom. Constr. 18, 1 (2008)

12. R. Volk, J. Stengel and F. Schultmann, Autom. Constr. 38 (2014)

13. M. Golparvar-Fard, J. Bohn, J. Teizer, S. Savarese and F. Peña-Mora, Autom. Constr. 20, 8 (2011)

14. A. Dimitrov and M. Golparvar-Fard, Adv. Eng. Inform. 28, 1 (2014)

15. X. Zhang, N. Bakis, T. C. Lukins, Y. M. Ibrahim, S. Wu, M. Kagioglou, G. Aouad, A. P. Kaka and E. Trucco, Autom. Constr. 18, 3 (2009)

16. K. K. Han and M. Golparvar-Fard, Autom. Constr. 53 (2015)

17. S. Kiziltas, A. Burcu, E. Ergen and T. Pingbo, ITcon 13. Special Issue Sensors in Construction and Infrastructure Management (2008)

18. J. Jung, S. Hong, S. Jeong, S. Kim, H. Cho, S. Hong and J. Heo, Autom. Constr. 42 (2014)

19. J. Yang, M.-W. Park, P. A. Vela and M. GolparvarFard, Adv. Eng. Inform. 29, 2 (2015)

20. D. H. Foster, K. Amano, S. M. C. Nascimento and M. J. Foster, J. Opt. Soc. Am. A Opt. Image Sci. Vis. 23, $10(2006)$

21. N. E. M. Nasarudin and H. Shafri, Journal of Urban and Environmental Engineering 5, 1 (2011)

22. M. Herold and D. Roberts, Appl. Opt. 44, 20 (2005)

23. V. C. Coffey, Opt. Photon. News 26, 10 (2015)

24. J. Behmann, J. Steinrücken and L. Plümer, ISPRS-J. Photogramm. Remote Sens. 93 (2014)

25. L. Granero-Montagud, C. Portalés, B. PastorCarbonell, E. Ribes-Gómez, A. Gutiérrez-Lucas, V. Tornari, V. Papadakis, R. M. Groves, B. Sirmacek, A. Bonazza, I. Ozga, J. Vermeiren, K. van der Zanden, M. Föster, P. Aswendt, A. Borreman, J. D. Ward, A. Cardoso, L. Aguiar, F. Alves, P. Ropret, J. M. LuzónNogué and C. Dietz, Proc. SPIE 8790, Optics for Arts, Architecture, and Archaeology IV, 879011 (2013)

26. E. J. Jaselskis, Z. Gao, A. Welch and D. O'Brien, Proceedings the Mid-Continent Transportation Research Symposium Iowa State University, Ames (2003)

27. S. Leutenegger, M. Chli and R. Y. Siegwart, Computer Vision (ICCV), 2011 IEEE International Conference on (2011)

28. H. Bay, A. Ess, T. Tuytelaars and L. Van Gool, Comput. Vis. Image Underst. 110, 3 (2008)

29. J. Matas, O. Chum, M. Urban and T. Pajdla, The British Machine Vision Conference, BMVA Press (2002)

30. A. Alahi, R. Ortiz and P. Vandergheynst, Computer Vision and Pattern Recognition (CVPR), 2012 IEEE Conference on (2012)
31. R. B. Rusu, Z. C. Marton, N. Blodow, M. Dolha and M. Beetz, Robot. Auton. Syst. 56, 11 (2008)

32. R. A. Schultz, T. Nielsen, J. R. Zavaleta, R. Runch, R. Wyatt and H. R. Garner, Cytometry 43, 4 (2001)

33. D. Connah, M. S. Drew and G. D. Finlayson, J. Opt. Soc. Am. A Opt. Image Sci. Vis. 32, 12 (2015)

34. L. Tamas and Z. Kato, Computer Vision Workshops (ICCVW), 2013 IEEE International Conference on (2013)

35. M. M. Darrodi, G. Finlayson, T. Goodman and M. Mackiewicz, J. Opt. Soc. Am. A Opt. Image Sci. Vis. 32, 3 (2015)

36. Z. Zhengyou, ITPAM 22, 11 (2000) 